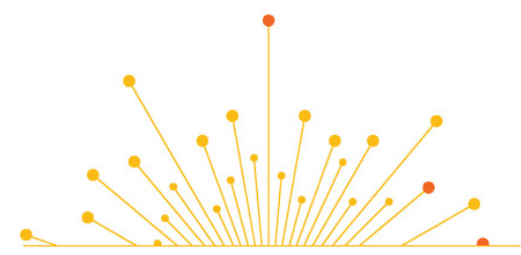

SCIENCE TABLE

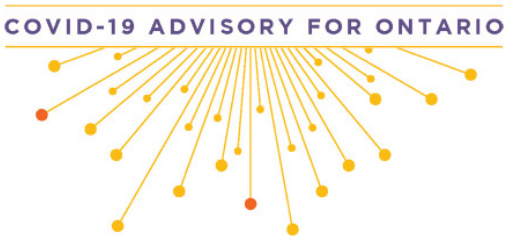

Version: $\mathbf{1 . 0}$

Published: August 26, 2021

Citation: Manuel DG, Delatolla R, Fisman $\mathrm{DN}$, et al. The role of wastewater testing for SARS-CoV-2 surveillance. Science Briefs of Ontario COVID-19 Science Advisory Table. 2021;2(40). https://doi.org/10.47326/ ocsat.2021.02.40.1.0

Author Affiliations: The affiliations of the members of the Ontario COVID-19 Science Advisory Table can be found at https:// covid19-sciencetable.ca/.

Declarations of Interest: The declarations of interest of the members of the Ontario COVID-19 Science Advisory Table, its Working Groups, or its partners can be found at https:// covid19-sciencetable.ca/. The declarations of interest of external authors can be found under additional resources at https://doi. org/10.47326/ocsat.2021.02.40.1.0

About Us: The Ontario COVID-19 Science Advisory Table is a group of scientific experts and health system leaders who evaluate and report on emerging evidence relevant to the COVID-19 pandemic, to inform Ontario's response. Our mandate is to provide weekly summaries of relevant scientific evidence for the COVID-19 Health Coordination Table of the Province of Ontario, integrating information from existing scientific tables, Ontario's universities and agencies, and the best global evidence. The Science Table summarizes its findings for the Health Coordination Table and the public in Science Briefs.

Correspondence to: Secretariat of the Ontario COVID-19 Science Advisory Table (info@covid19-sciencetable.ca)

Copyright: 2021 Ontario COVID-19 Science Advisory Table. This is an open access document distributed under the terms of the Creative Commons Attribution License, which permits unrestricted use, distribution, and reproduction in any medium, provided that the original work is properly cited.

The views and findings expressed in this Science Brief are those of the authors and do not necessarily reflect the views of all of the members of the Ontario COVID-19 Science Advisory Table, its Working Groups, and its partners.
SCIENCE BRIEFS

\section{The Role of Wastewater Testing for SARS-CoV-2 Surveillance}

Douglas G. Manuel, Robert Delatolla, David N. Fisman, Meghan Fuzzen, Tyson Graber, Gabrielle M. Katz, JinHee Kim, Chrystal Landgraff, Alex MacKenzie, Antonina Maltsev, Anna Majury, R. Michael McKay, John Minnery, Mark Servos, J. Scott Weese, Allison McGeer, Karen B. Born, Kali Barrett, Brian Schwartz, Peter Jüni on behalf of the Ontario COVID-19 Science Advisory Table and the Wastewater Surveillance Science and Implementation Tables

\section{Key Message}

Wastewater testing for SARS-CoV-2 is relatively new; however, it builds on existing public health surveillance infrastructure. There is a limited but growing evidence base for its use, despite notable interpretation challenges. Wastewater testing results have helped to inform public health policy and interventions during the COVID-19 pandemic in Ontario and other jurisdictions. Wastewater testing for SARS-CoV-2 is useful for early detection of outbreaks and surges as well as population-wide surveillance of COVID-19 that is complementary to clinical testing. Further, it offers an efficient means of SARS-CoV-2 surveillance for specific settings such as correctional facilities, shelters, and university residences. Wastewater testing can also be used for the detection and monitoring of variants of concern (VOCs).

\section{Summary}

\section{Background}

Wastewater testing has a history of informing public health action through its use to monitor health threats such as polio, antimicrobial resistance, and illicit drug use in populations. It can be used to detect components of SARS-CoV-2.

SARS-CoV-2 has characteristics which pose a challenge for public health surveillance approaches. These include a high rate of transmission by symptomatic, asymptomatic, and pre-symptomatic individuals that leads to missed case detection and an interval between viral transmission to clinical testing that leads to delays in case detection. These characteristics of SARS-CoV- 2 infection, along with the observation that SARSCoV-2 is excreted in stools during all phases of infection, has led to the uptake of wastewater testing to complement SARS-CoV-2 surveillance based on clinical tests and case identification.

\section{Questions}

What are the current and future applications of SARS-CoV-2 wastewater testing for SARS-CoV-2 surveillance?

What is required to ensure wastewater testing for SARS-CoV-2 provides accurate, reliable, and actionable information that informs public health interventions for the mitigation and management of the COVID-19 pandemic?

What are the international and Ontario experiences with wastewater testing?

\section{Findings}

Evidence of wastewater testing for SARS-CoV-2 to inform a public health response is rapidly evolving, though is currently limited to case reports. In Ontario and other 
jurisdictions, the main benefit to date has been the identification of new COVID-19 outbreaks in specific populations in advance of other surveillance methods. However, the more common use for wastewater testing is to monitor the prevalence of SARSCoV-2 - a complementary approach to clinical testing. Early outbreak detection has led to enhanced public health measures and informed communication with the public regarding COVID-19 cases and the potential risk of increased transmission.

Wastewater testing is not without challenges. Three issues need further consideration to ensure wastewater testing reliably informs COVID-19 public health interventions.

1. Analytic methods: approaches for sampling and measuring viral RNA vary considerably across jurisdictions, as do the populations surveyed and the sewer networks. There is a need for improved quality assurance between analytical methods, as well as consistency in data interpretation within local context.

2. Surveillance programs: most wastewater testing programs worldwide are still in the developmental stages, and while capacity is expanding, there is need to transition to a sustainable system.

3. Collaboration: public health, testing laboratories, and wastewater utilities need to collaborate to ensure testing provides actionable public health intelligence which is interpreted and communicated and actioned effectively.

Wastewater testing for COVID-19 has rapidly expanded worldwide with over 2,200 testing sites in 55 countries. The Canadian Water Network organizes the COVID-19 Wastewater Coalition, bringing together municipal utilities, researchers, public health agencies, and governments. Currently, wastewater testing for SARS-CoV-2 is performed at over 200 sites in Canada, including all provinces and two territories. Ontario launched a \$12 million province-wide Wastewater Surveillance Initiative in fall 2020 led by the Ministry of Environment, Conservation and Parks. As of August 2021, all 34 public health units have joined the initiative with approximately 160 sampling locations covering over $80 \%$ of the population. Additional testing is performed by the National Microbiology Laboratory, research initiatives such as Coronavirus Variants Rapid Response Network (CoVaRR-Net) and Ontario Genomics, and university-led programs. In addition to municipal level testing, these programs perform testing in select Indigenous communities, university campuses, correctional facilities, shelters, hospitals, retirement homes, neighbourhoods, and long-term care (LTC) facilities.

\section{Interpretation}

Wastewater testing addresses several key challenges for SARS-CoV-2 surveillance. As a surveillance approach, wastewater testing includes anyone who excretes SARSCoV-2, whether symptomatic, asymptomatic, or pre-symptomatic, thereby potentially reducing the lag time between SARS-CoV-2 infection and surveillance reporting. A single wastewater test can measure the level of SARS-CoV-2 infection for entire populations. Wastewater testing has informed public health policy and interventions in Ontario and international jurisdictions. However, there is likely publication bias, with favourable case reports being more likely than remaining reports to be published, and there are few reports with control populations. Wastewater testing as a surveillance tool for SARS-CoV-2 is relatively new and requires ongoing development, standardization, capacity building, and sustainable infrastructure.

\section{Background}

Surveillance of SARS-CoV-2 can be a challenge due to transmission by symptomatic, and pre-symptomatic individuals. These unique transmission characteristics can lead to missed case detection and delays in case detection. ${ }^{1-3}$ Seroprevalence studies have suggested that the majority of people infected with SARS-CoV-2 go unreported. ${ }^{4-6}$ 
Wastewater testing can be used to detect components of SARS-CoV-2. Wastewater testing has been used for other forms of public health surveillance, including detection and monitoring of pollutants (e.g., mercury) and biomarkers, and to obtain data on the activities and health of the community living within the catchment area. Wastewater testing has been previously used to detect illicit drug use as well as the presence of viruses (e.g., poliovirus) within a community and for surveillance of serious health threats such as polio, antimicrobial resistance, and illicit drug use in populations. ${ }^{1,7}$

Research into the dynamics of SARS-CoV-2 RNA fecal shedding is at an early stage. SARS-CoV-2 RNA is detectable in stool samples of approximately 30 to $60 \%$ of cases. ${ }^{8-11}$ Fecal shedding of detectable viral RNA can persist for up to 45 days in symptomatic cases, with a median of 22 days (interquartile range (IQR): 17 to 31). ${ }^{10,12,13}$ There is little to no difference in stool shedding based on disease severity, ${ }^{14,15}$ and children and adults are equally likely to shed SARS-CoV-2 RNA in their stool. ${ }^{16}$ Most studies exploring shedding dynamics have focused on participants with a confirmed diagnosis of COVID-19 with most stool samples collected after symptom onset. ${ }^{9,12,14,15,17,18} \mathrm{~A}$ recent study investigating fecal shedding during a COVID-19 dormitory outbreak identified that $70 \%$ of infected students with fecal shedding of SARS-CoV-2 RNA were asymptomatic. ${ }^{19}$

\section{Questions}

What are the current and future applications of SARS-CoV-2 wastewater testing for SARS-CoV-2 surveillance?

What is required to ensure wastewater testing for SARS-CoV-2 provides accurate, reliable, and actionable information that informs public health interventions for the mitigation and management of the COVID-19 pandemic?

What are the international and Ontario experiences with wastewater testing?

\section{Findings}

\section{Current and Future Applications of Wastewater Testing for SARS-CoV-2 Surveillance}

Current uses for wastewater testing for SARS-CoV-2 include early detection of outbreaks and surges, specific population surveillance, and surveillance of VOCs.,20,21 Potential future uses include later-stage surveillance and leveraging infrastructure to monitor other pathogens and health risks. ${ }^{22}$

\section{Early Detection of Outbreaks and Surges}

Wastewater testing has been shown to identify SARS-CoV-2 RNA in advance of laboratory-based clinical confirmation of COVID-19. In Northern Italy, the first SARS-CoV-2 positive wastewater samples were found several weeks before the first imported COVID-19 cases were reported. ${ }^{23}$ Studies from the Netherlands show a linear correlation between the concentration of viral RNA copies in wastewater and the cumulative number of active COVID-19 cases reported in six cities in March 2020. ${ }^{24}$

Canadian reports of SARS-CoV-2 detection through wastewater testing prior to case detection through clinical testing occurred in Yellowknife, Northwest Territories in December 2020. Following positive wastewater tests, clinical testing was expanded, and recent travellers were encouraged to be tested. Clinical testing identified five cases in recent travellers who were at the end of their self-isolation period (Figure 1). No additional SARS-CoV-2 cases were reported, and SARS-CoV-2 RNA in wastewater dropped to undetectable levels.

There have been additional public reports in Canada and in other jurisdictions of positive wastewater tests in rural and remote communities in the setting of no clinical cases. ${ }^{25,26}$ This can inform notification and engagement of rural and remote communities. 
However, there is significant variation in the sensitivity and lead time for wastewater testing and reporting across Canadian jurisdictions and internationally. Further, the lack of systematic reviews to date raises concerns around publication bias around the frequency of positive wastewater tests in regions with no clinical cases. ${ }^{27}$

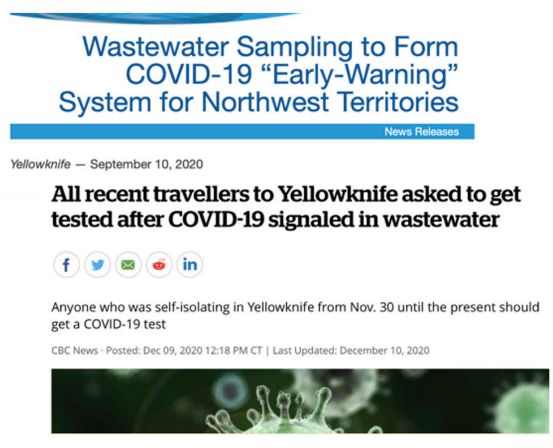

Dec. 9, 2020

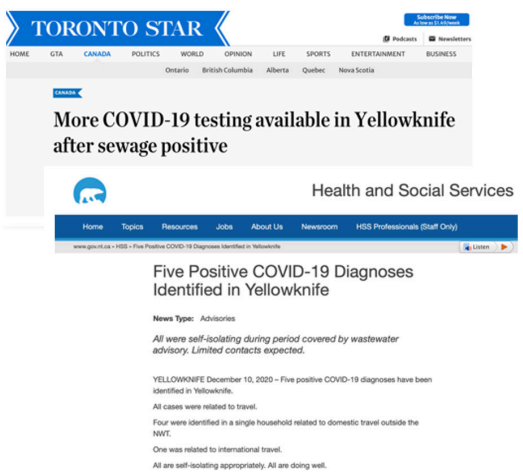

Dec. 10, 2020

Figure 1. Newspaper Headlines from Yellowknife, Northwest Territories from December 9 and December 10, 2020 Newspaper headlines describing positive wastewater tests and the policy response. ${ }^{28}$

\section{Population-Wide Surveillance}

Numerous dashboards that display wastewater tests alongside clinical tests have emerged worldwide, including in Canada. ${ }^{29}$ Typically, wastewater test results are presented as time trends to inform the pattern of "waves" or surges of increasing and decreasing cases. For example, Ottawa Public Health uses wastewater testing to support identification of new COVID-19 surges with early notification to public health and the population through social media, news conferences, and public reporting (Figure 2).

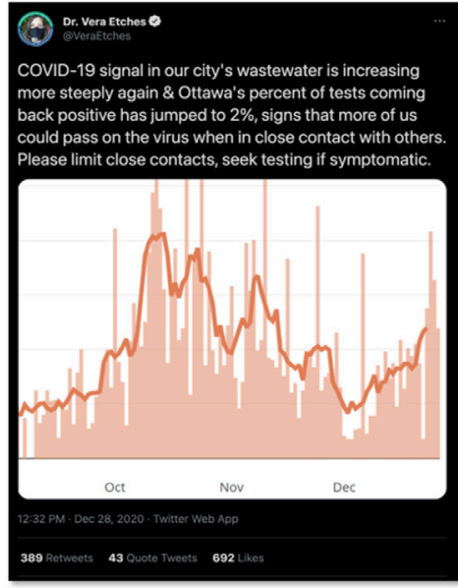

4
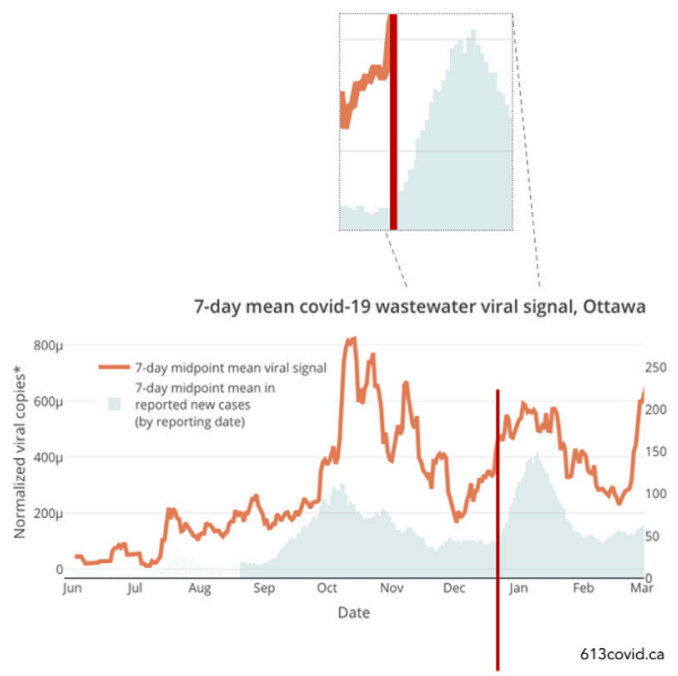

Dec. 28, 2020

Figure 2. Early Notification Wastewater Testing Results in Ottawa on December 28, 2020

Ottawa is an example of a Canadian municipality that includes public wastewater testing to inform and engage residents in prevention and control measures. The city's medical officer of health, Dr. Vera Etches, commonly includes wastewater testing in her public messages (Figure 2).

\section{Specific Population Surveillance}

The World Health Organization (WHO) and others have highlighted the use and benefits of wastewater testing in high-risk residential settings, such as LTC homes, correctional facilities, and worker dormitories.,21,30 For example, wastewater testing 
was implemented at residential housing for approximately 5,000 University of Arizona students in August 2020. When there was SARS-CoV-2 RNA detected in one dormitory's wastewater, further clinical testing of all building residents identified two positive asymptomatic cases who were isolated, resulting in no further transmission. ${ }^{31-33}$

Wastewater testing has been identified as a surveillance approach for non-residential facilities such as schools or office buildings, ${ }^{21}$ albeit they will be constrained by the defecation frequency during office hours and other factors. ${ }^{34-39}$ Sampling and interpretation difficulties may arise alongside social implications (e.g., stigmatization of vulnerable communities) of wastewater testing at local and regional levels.,40

\section{Variants and Mutations of Interest and Concern}

Wastewater surveillance can assess the prevalence of VOCs. There are typically two steps or approaches in clinical samples. First, variants can be screened or identified using clinical PCR testing. PCR tests for variants examine either mutations that are present in one or more VOC or RNA sequences for a specific variant. Second, RNA from a given sample may be fully sequenced to confirm the specific type(s) of known variant(s) and potentially detect new mutations. Different jurisdictions utilize varying levels of VOC screening and sequencing contingent on the testing and sequencing resources available. Sequencing can be performed on a population sample or on a subset of screen positive tests that represent particular concerns, such as travellers, those with severe illness, or high viral load.

VOC assessment using wastewater testing applies approaches similar to clinical testing with a strength for estimating population prevalence. First, PCR primers can be designed to identify specific VOCs. ${ }^{41}$ Second, a near-complete SARS-CoV-2 genomic sequences can be determined from using metagenome assays, also known as the consensus community genome. ${ }^{41}$ In this fashion, the proportion of different VOCs can be estimated (Figure 3 ) in addition to the regular assessment of the number of viral copies (Figure 4).

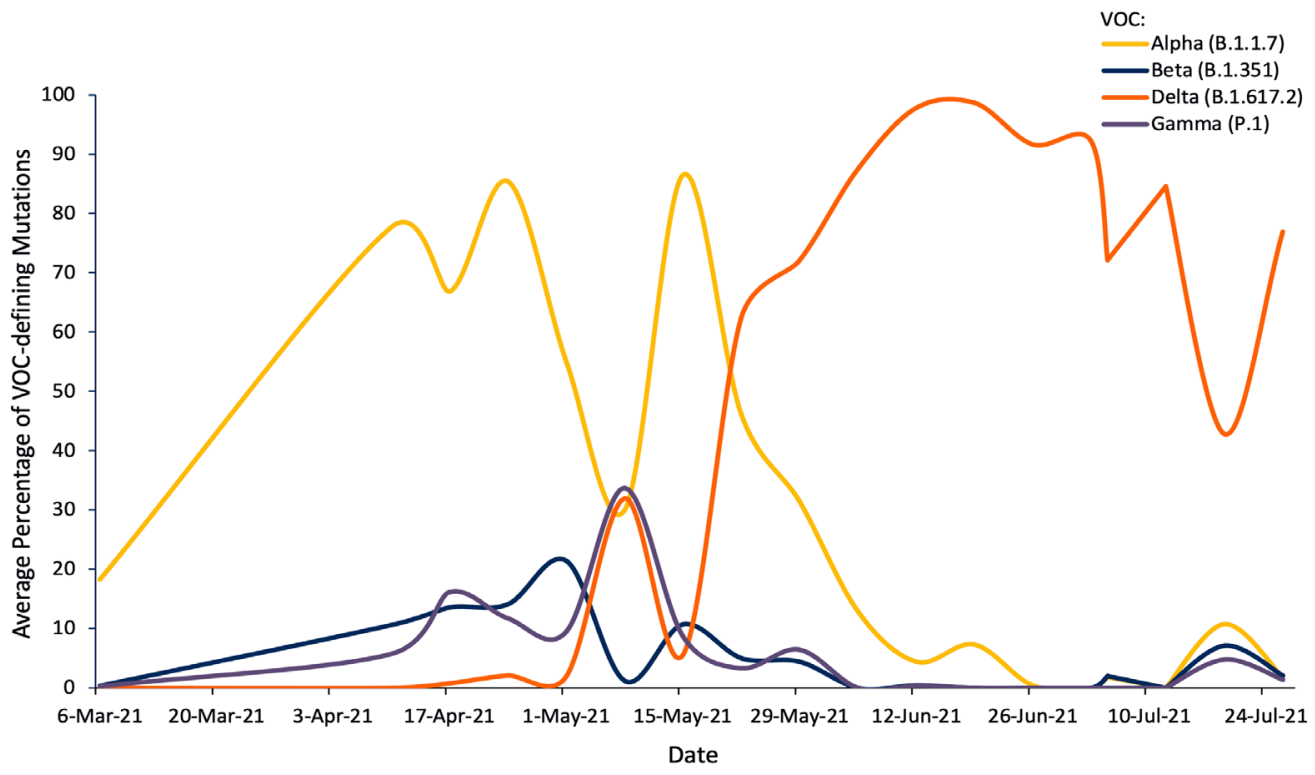

Figure 3. Percentage of VOC-Defining Mutations in Sequenced Samples from Kitchener, Ontario

Figure presenting the average percentages of VOC-defining mutations for the alpha, beta, delta, and gamma variants in samples collected between March 6 and July 26, 2021. These data were obtained by sequencing samples collected from wastewater in Kitchener, Ontario. Data sourced from personal correspondence with Chrystal Landgraff. 


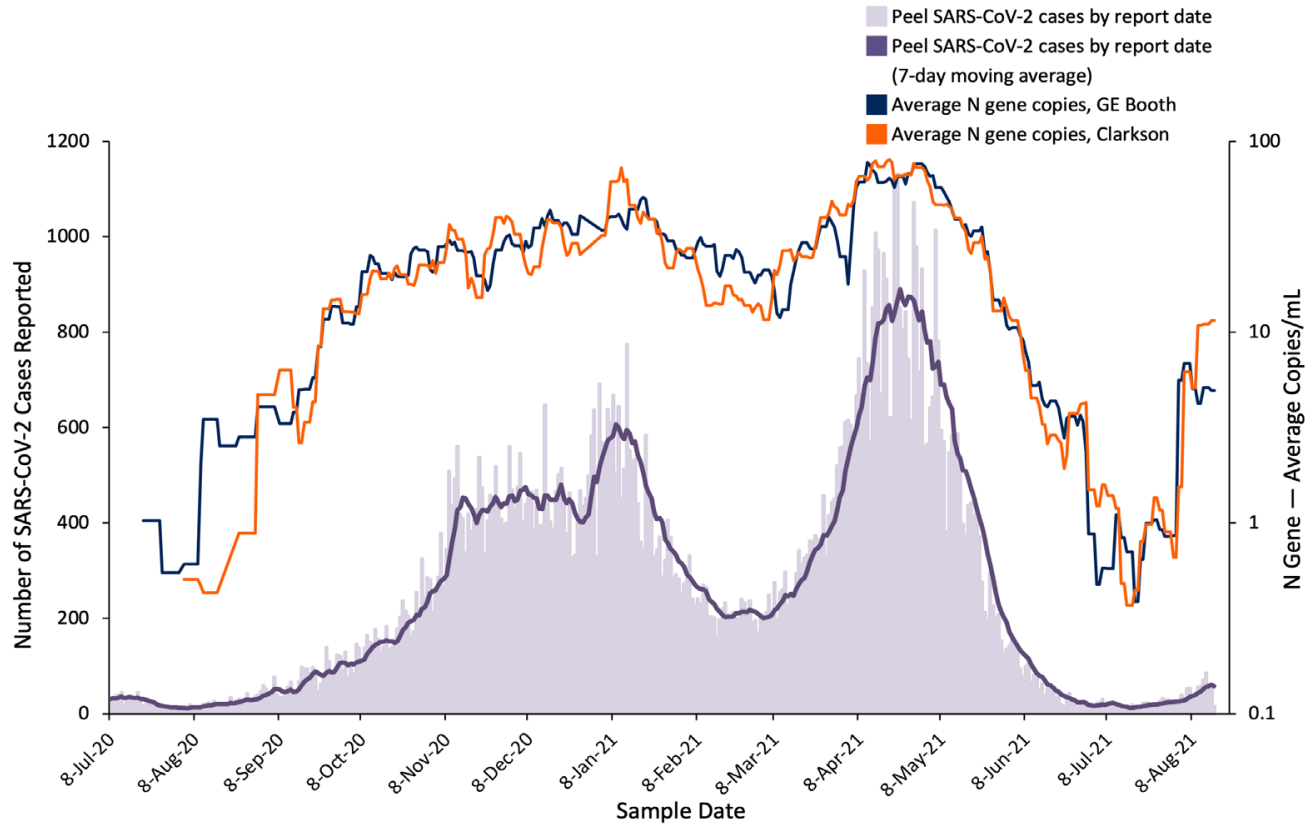

Figure 4. SARS-CoV-2 Cases and Average Viral N Gene Copies/mL in Samples from Wastewater in Peel Region Figure presenting new SARS-CoV-2 cases reported in Peel Region and the 7-day moving average, as well as 7-day moving averages of copies/mL of viral $\mathrm{N}$ gene in samples from two Peel Region wastewater treatment plans. Data from July 8, 2020 to August 15, 2021 are included in this figure. N gene copies are a marker for SARS-CoV-2. The average copies/ $\mathrm{mL}$ of $\mathrm{N}$ gene is presented using a logarithmic scale. Data sourced from personal correspondence with Mark Servos.

\section{Later-Stage Monitoring}

As with other infectious diseases, sustained vigilance in surveillance can be challenging as transmission ebbs. Wastewater testing has been proposed for ongoing surveillance in the late stages of the COVID-19 pandemic, in low-risk populations, and for ongoing monitoring as SARS-CoV-2 becomes endemic.

\section{Monitoring of Other Pathogens and Health Risks}

The development of surveillance infrastructure may scale to other public health concerns, including pathogens that are excreted in feces such as influenza, antibiotic resistant bacteria, food borne pathogens as well as illicit drugs. ${ }^{2,42,43}$

\section{Requirements to Ensure Wastewater Testing for SARS-CoV-2 Provides Reliable, Accurate and Actionable Information}

The WHO, the Centers for Disease Control and Prevention (CDC), the Public Health Agency of Canada (PHAC), and others have identified analytic methods, reporting, and interpretation of tests and program sustainability as three areas that require additional development. ${ }^{2,20,21,44}$

\section{Analytic Methods to Measure SARS-CoV-2 in Wastewater}

Wastewater testing for pathogens such as polio have been developed over several decades. ${ }^{2}$ However, analytic methods vary considerably across jurisdictions - as do the populations surveyed and the wastewater infrastructure. The COVID-19 pandemic has spurred rapid expansion in wastewater testing. Many quality assurance challenges remain; however, multiple factors influence the quantity of viral material in wastewater and thus the quantity of reported RNA measured in any given testing site and between testing sites. . $22,45,46^{-1}$ 
The factors that affect test results are:

Variations between people

- The level of RNA excreted in stools rapidly increases during early illness, then plateaus before decreasing. Wastewater testing captures excreted RNA during all these phases with a single person's stool RNA entering the sewage system over several days or weeks. As discussed earlier, the overall level of RNA excreted or entering the wastewater system varies depending on age, fecal excretion patterns, and other individual characteristics.

Variations in wastewater infrastructure and the environment

- There are differences in the transit time and quantity of RNA that remains intact between an individual's stool and the sample collection point. These differences arise from variation in the size and design of sewershed, rainfall draining into combined sewers, the use of holding tanks and pumping stations, the fragility of the viral RNA, and factors such as temperature or substances that affect the integrity of the viral RNA.

Variations in sample collection and method of measuring SARS-CoV-2 viral load

- Wastewater samples can be collected from free-flowing street sewage utility holes, influent, or primary sludge in wastewater treatment plants, among other settings. There is an increasing number of sampling approaches including grab sampling, passive samplers, and autosamplers, each with their own advantages and limitations. There are also different approaches to transport, store and concentrate a sample prior to RNA extraction and measurement. Measurement of SARS-CoV-2 is largely performed in Canada and across the world using two types of PCR (RT-qPCR or RT-d(d)PCR) with similar primers; however, there are distinct calibration, quality assurance, and standardization approaches.

- Quality assurance and inter-laboratory studies can be used to compare different analytical methods to ensure comparability and consistency. ${ }^{47,48}$

- Despite concerns about many sources of test variation, wastewater test sensitivity appears high. Evidence of high sensitivity is supported from an increasing number of case studies which detected SARS-CoV-2 in wastewater prior to clinical tests, and subsequently confirmed SARS-CoV-2 through clinical testing. ${ }^{12,19}$ However, the sensitivity of case detection in populations is not clearly understood and is assumed to vary between settings. ${ }^{3,25}$ The case detection sensitivities in the dormitory and other congregate setting outbreaks described in this report range from 1 to 10 cases per 1,000 residents. ${ }^{3,21,30,32,33,49} \mathrm{~A}$ study of 353 community sites estimated an $80 \%$ rate of detection of SARS-CoV-2 in wastewater at a clinical incidence of 13 cases per 100,000 people. ${ }^{50}$ Hart and Halden estimated that one infected individual theoretically is detectable in wastewater among 100 to 2,000,000 persons. ${ }^{51}$

Several studies examined those factors that influence the specificity and reliability of test results. To date, these studies have not identified factors with a strong influence on test performance. ${ }^{2,42,43}$ Apart from small studies, ascertaining the true number of COVID-19 cases for test performance studies has been challenging. Comparing the results of wastewater testing from municipal wastewater treatment plants to clinically reported cases is discouraged because clinical cases are frequently not diagnosed and the level of testing can vary over time. ${ }^{2}$

\section{Reporting and Interpreting Wastewater Tests}

The optimal means of reporting wastewater testing data is still evolving. Common reporting approaches use averages to smooth day-to-day variability. ${ }^{20,45}$ Environmental 
changes are addressed using standardization and normalization approaches, including various controls to account for the variable dilution of human waste inherent to all wastewater. ${ }^{52}$

Interpretation involves leveraging collaborative expertise at the local level where testing is performed. ${ }^{2,20,21,53}$ This would include local public health authorities, municipal wastewater utilities, and testing laboratories. Under this paradigm, public health staff interpret wastewater tests along with traditional SARS-CoV-2 surveillance metrics. They also develop and implement a public health response considering the likelihood of further transmission, available resources, and intervention effectiveness. Staff of municipal wastewater utilities understand the wastewater system and factors that potentially affect RNA transit time and viability. Wastewater engineers and scientists understand assay performance characteristics and the relationship between the wastewater systems, sample collection, and reported virus levels. Many jurisdictions report challenges developing multidisciplinary approach to interpretation, response, and community guidance given the scale and complexity of issues. ${ }^{2,53}$

The first detection of SARS-CoV-2 in Yellowknife wastewater in December 2020 is an example of how public health authorities can consider different responses based on the likelihood of ongoing SARS-CoV-2 transmission. Directly following SARS-CoV-2 detection, there was a public announcement of the wastewater test result and a recommendation that all recent travellers be tested. This recommendation was made because of concerns that not all recent travellers were compliant with the requirement to self-quarantine for two weeks. Other municipalities with a first detection of SARSCoV-2 during periods with high levels of restrictions have provided a public notice without recommendation for enhanced clinical testing because they have assessed further SARS-CoV-2 transmission as low.

\section{Development of Sustained Surveillance Programs}

With the declaration of a global pandemic in March 2020, wastewater surveillance was rapidly launched in many jurisdictions such as the Netherlands. ${ }^{24,29}$ Testing expanded rapidly from academic pilot projects to emerging regional and national programs. In jurisdictions where such a transition to ongoing state funding does not occur, the sustainability of programs, reliant on short-term or research funding, is unclear.

The European Commission (EC) has instituted wastewater testing as part of the Member State surveillance program under the Health Emergency Preparedness and Response Authority (HERA) Incubator program for variant surveillance. ${ }^{53,54}$ Throughout the European Union, there are 600 sites planned by end of July 2021, with 6,000 sites planned for a fully-implemented system. ${ }^{55}$ The United States has incorporated SARS-CoV-2 testing into the National Wastewater Surveillance System. ${ }^{20}$ The United States also announced national funding for wastewater assessment of VOCs in 320 sites covering 100 million people. ${ }^{56}$ In Australia and New Zealand, the Collaboration on Sewage Surveillance of SARS-COV-2 (ColoSSoS) Project is integrating wastewater testing data with clinical surveillance data. ${ }^{57}$

The Public Health Agency of Canada (PHAC) coordinates wastewater surveillance activity with the National Microbiology Laboratory (NML), providing testing in communities in Saskatchewan, Yukon, and the Northwest Territories. ${ }^{58}$ The NML also performs VOC sequencing in cities across Canada. Québec, Alberta, and British Columbia have programs with provincial coordination. Currently, the Ontario Wastewater Surveillance Initiative is funded until March 2022. The funding timeline for other wastewater testing programs in Canada is unclear.

\section{The Ontario Experience with Wastewater Testing}

Case examples, in which wastewater testing in Ontario has informed public health 
action in each of the four uses previously described, include the following:

Early detection of outbreaks and surges

On July 17, 2020, the city of Ottawa detected SARS-CoV-2 in wastewater, an early indication which motivated the transition to daily testing and reporting wastewater testing to Ottawa Public Health. Provincial stay at home orders were issued in the City on December 26, 2020 in consideration of a two week early rise in wastewater signal prior to the corresponding increase in clinical cases. ${ }^{59}$

Wastewater testing resulted in early detection of transmission that led to increased testing or public health measures also in other settings, including university residences and small communities. For example, at the University of Windsor's Alumni Hall residence, a positive wastewater test was reported on March 27, 2021. Testing was performed for 195 people over two days. Two asymptomatic cases were identified, which resulted in isolation of the cases and enhanced public health measures in the affected area. Wastewater tests identified the Alpha (B.1.1.7) variant, which was confirmed one week later in the clinical tests in the two asymptomatic cases. ${ }^{49}$

\section{Population Surveillance of Transmission}

Local public health bodies are in the early stages of incorporating wastewater results into their surveillance programs. Samples are collected from wastewater treatment plants across Ontario. $80 \%$ of the population is covered by the Wastewater Surveillance Initiative (Carrasco S, personal communication). Five Ontario municipalities publicly report wastewater test results, and three others include wastewater results in their regular public briefing. In addition, there are weekly federal and Ontario meetings for municipalities and wastewater testing laboratories to share experiences, including best practices and interpretive challenges. Public Health Ontario has provided a summary of global wastewater surveillance activities and considerations for assessing and interpreting test data. ${ }^{60}$

Ottawa is a leading Canadian municipality for integrating wastewater testing into COVID-19 prevention and control response. Public reporting of wastewater testing began in October 2020 and was added to a widely-viewed website alongside projections, case numbers, and hospitalization data (https://613covid.ca).

\section{Specific Population Surveillance}

In Ontario, wastewater testing is currently performed in 77 upstream locations (i.e., in sites upstream to wastewater plants), including 19 university campus locations, five retirement homes, four long-term care homes, three correctional facilities, three shelters, and two hospitals. There is also community-level testing in several Indigenous communities. There have been several case reports of wastewater results informing public health prevention and control, such as at the University of Windsor.

\section{Variants of Interest and Concern}

Ontario began wastewater testing for variants in January 2021 using the PCR method to identify the Alpha (B.1.1.7) VOC and has since expanded through a Genomics Ontario program. ${ }^{61}$ The National Microbiology Laboratory is using the metagenomic sequencing method to test wastewater in select municipalities across Canada, including Ontario.41 In Ottawa, the National Microbiology Laboratory has detected the Delta (B.1.617.2) variant in wastewater since April 2021.

\section{Interpretation}

Wastewater testing for SARS-CoV-2 is a rapidly developing surveillance approach. Evidence of the effectiveness of wastewater testing in informing public health action 
to reduce SARS-CoV-2 transmission is accumulating. There is an increasing number of case reports where wastewater testing has informed public health policy through early detection of surges in community SARS-CoV-2 transmission, and assessment of VOC prevalence. As COVID-19 becomes endemic, wastewater testing may be useful for ongoing surveillance in low-risk populations. Wastewater testing has a number of benefits, including an ability to sample a wide population and to access settings where testing may be limited, as well as relative cost effectiveness compared to other SARS-CoV-2 testing approaches. Limitations include inability to test water systems that are not connected to a wastewater system such as septic tanks, differences in measurement, sample and data collection modalities, and need for more evidence to inform thresholds for implementation of public health interventions.

Wastewater testing includes the entire population serviced by the sampled wastewater infrastructure. Populations which may have reduced access to clinical testing are included in wastewater testing. Furthermore, underserviced and vulnerable populations can be specifically targeted by collecting wastewater from neighbourhoods or congregate settings with a higher risk of SARS-CoV-2 infection and where there may be barriers to accessing clinical testing. ${ }^{62}$

Nevertheless, populations not served by sewer networks and centralized infrastructure may be missed by wastewater testing. These include populations at high risk of transmission (e.g., migrant farm workers) or high risk of disease complications (e.g., LTC residents). LTC residents and other individuals may not contribute to municipal wastewater collection due to use of incontinence apparel. Migrant farm workers may live in bunk houses that use septic fields. Some northern and remote communities lack municipal systems and collect waste through sewage trucks or decentralized septic tanks. In the Northwest Territories, sewage testing programs included sampling from sewage trucks. ${ }^{63}$

Wastewater testing is likely to be cost-effective compared to other SARS-CoV-2 surveillance systems. A hypothetical scenario estimated that 230 million people in the United States ( $70 \%$ of the population) could be covered by analyzing all 15,014 existing wastewater treatment plants with the cost of reagents being approximately 225,000 USD. ${ }^{64}$ If the 230 million people were tested once individually, the estimated cost of reagents was estimated at 2.45 billion USD. That stated, there is no current consensus on population size and testing frequency for wastewater testing or clinical tests.

Further, the personnel required for wastewater testing are different from the personnel for clinical testing and leverage existing municipal wastewater staff and academic or commercial wastewater testing labs. Fewer resources and clinical staff for wastewater testing compared to clinical testing may be an important consideration in low-resource settings that lack sufficient clinical staff.

\section{Methods used for this Science Brief}

We searched PubMed, Google Scholar, the Joanna Briggs Institute's COVID-19 Special Collection, LitCovid in PubMed, the Oxford COVID-19 Evidence Service, the World Health Organization's Global Literature on Coronavirus Disease, and other COVID-19 specific resources listed by the Guidelines International Network and the McMaster Health Forum. In addition, we retrieved reports citing relevant articles through Google Scholar and reviewed references from identified articles for additional studies. Details of the Ontario Wastewater Initiative were provided by the Ministry of the Environment, Conservation and Parks.

\section{Author Contributions}

DGM and PJ conceived the Science Brief and wrote the first draft. All authors revised 
the Science Brief critically for important intellectual content and approved the final version.

\section{References}

1. Asghar H, Diop OM, Weldegebriel G, et al. Environmental surveillance for polioviruses in the Global Polio Eradication Initiative. J Infect Dis. 2014;210 Suppl 1:S294-303. https://doi.org/10.1093/infdis/jiu384

2. World Health Organization. Rapid Expert Consultation on Environmental Surveillance of SARS-COV-2 in Wastewater. World Health Organization; 2020:17. https://apps.who.int/iris/bitstream/handle/10665/334305/WHOEURO-2020\%20-1093-40839-55199-eng.pdf

3. World Health Organization. Status of Environmental Surveillance for SARS-CoV-2 Virus.; 2020. https://www.who.int/news-room/commentaries/detail/status-ofenvironmental-surveillance-for-sars-cov-2-virus

4. Public Health Ontario. COVID-19Seroprevalence in Ontario: March 27, 2020 to June 30, 2020.; 2020:10. https://www.publichealthontario.ca/-/media/documents/ ncov/epi/2020/07/covid-19-epi-seroprevalence-in-ontario.pdf?la=en

5. Stringhini S, Wisniak A, Piumatti G, et al. Seroprevalence of anti-SARS-CoV-2 IgG antibodies in Geneva, Switzerland (SEROCoV-POP): a population-based study. The Lancet. 2020;0. https://doi.org/10.1016/S0140-6736(20)31304-0

6. Sood N, Simon P, Ebner P, et al. Seroprevalence of SARS-CoV-2-specific antibodies among adults in Los Angeles County, California, on April 10-11, 2020. JAMA. Published online May 18, 2020. https://doi.org/10.1001/jama.2020.8279

7. O'Reilly KM, Allen DJ, Fine $\mathrm{P}$, Asghar $\mathrm{H}$. The challenges of informative wastewater sampling for SARS-CoV-2 must be met: lessons from polio eradication. Lancet Microbe. 2020;1(5):e189-e190. https://doi.org/10.1016/S2666-5247(20)30100-2

8. Wang $\mathrm{W}, \mathrm{Xu}, \mathrm{Y}, \mathrm{Gao} \mathrm{R}$, et al. Detection of SARS-CoV-2 in Different Types of Clinical Specimens. JAMA. 2020;323(18):1843-1844. https://doi.org/10.1001/ jama.2020.3786

9. Pan Y, Zhang D, Yang P, Poon LLM, Wang Q. Viral load of SARS-CoV-2 in clinical samples. Lancet Infect Dis. 2020;20(4):411-412. https://doi.org/10.1016/S14733099(20)30113-4

10. Cheung KS, Hung IFN, Chan PPY, et al. Gastrointestinal manifestations of SARSCoV-2 infection and virus load in fecal samples from a Hong Kong cohort: systematic review and meta-analysis. Gastroenterology. 2020;159(1):81-95. https://doi. org/10.1053/j.gastro.2020.03.065

11. Jones DL, Baluja MQ, Graham DW, et al. Shedding of SARS-CoV-2 in feces and urine and its potential role in person-to-person transmission and the environmentbased spread of COVID-19. Sci Total Environ. 2020;749:141364. https://doi. org/10.1016/j.scitotenv.2020.141364

12. Zhang W, Du R-H, Li B, et al. Molecular and serological investigation of 2019-nCoV infected patients: implication of multiple shedding routes. Emerg Microbes Infect. 2020;9(1):386-389. https://doi.org/10.1080/22221751.2020.1729071

13. Stanoeva KR, Eijk AA van der, Meijer A, Kortbeek LM, Koopmans MPG, Reusken CBEM. Towards a sensitive and accurate interpretation of molecular testing for SARS-CoV-2: a rapid review of 264 studies. Eurosurveillance. 2021;26(10):2001134. https://doi.org/10.2807/1560-7917.ES.2021.26.10.2001134

14. Zheng S, Fan J, Yu F, et al. Viral load dynamics and disease severity in patients 
infected with SARS-CoV-2 in Zhejiang province, China, January-March 2020: retrospective cohort study. BMJ. 2020;369:m1443. https://doi.org/10.1136/bmj. m1443

15. Han MS, Seong M-W, Kim N, et al. Viral RNA load in mildly symptomatic and asymptomatic children with COVID-19, Seoul. Emerg Infect Dis. 2020;26(10). https://doi.org/10.3201/eid2610.202449

16. Singer A, Wray R. Detection and survival of SARS-coronavirus in human stool, urine, wastewater and sludge. Published online June 18, 2020. https://doi. org/10.20944/preprints202006.0216.v2

17. Lo IL, Lio CF, Cheong HH, et al. Evaluation of SARS-CoV-2 RNA shedding in clinical specimens and clinical characteristics of 10 patients with COVID-19 in Macau. Int J Biol Sci. 2020;16(10):1698. https://doi.org/10.7150/ijbs.45357

18. Wölfel R, Corman VM, Guggemos W, et al. Virological assessment of hospitalized patients with COVID-2019. Nature. 2020;581(7809):465-469. https://doi. org/10.1038/s41586-020-2196-x

19. Schmitz BW, Innes GK, Prasek SM, et al. Enumerating asymptomatic COVID-19 cases and estimating SARS-CoV-2 fecal shedding rates via wastewaterbased epidemiology. medRxiv. Published online April 18, 2021. https://doi. org/10.1101/2021.04.16.21255638

20. Centers for Disease Prevention and Control. National Wastewater Surveillance System (NWSS). Accessed March 24, 2021. https://www.cdc.gov/healthywater/ surveillance/wastewater-surveillance/wastewater-surveillance.html?CDC_AA_ refVal=https\%3A\%2F\%2Fwww.cdc.gov\%2Fcoronavirus\%2F2019-ncov\%2Fcasesupdates\%2Fwastewater-surveillance.html

21. Public Health Agency of Canada. SARS-CoV-2 wastewater-based surveillance: a guide for Public Health Action. Published March 2021. https://docs.google.com/ document/d/1jW_el63LSZDO60MEyul3ryFGLNVCcqNCzbR5IfObMa8/edit

22. Keshaviah A, HuXC, Henry M. Developing a flexible national wastewater surveillance system for COVID-19 and beyond. Environ Health Perspect. 2021;129(4). https:// doi.org/10.1289/EHP8572

23. Rosa GL, Mancini P, Ferraro GB, et al. SARS-CoV-2 has been circulating in northern Italy since December 2019: evidence from environmental monitoring. medRxiv. Published online June 26, 2020:2020.06.25.20140061. https://doi. org/10.1101/2020.06.25.20140061

24. Medema G, Heijnen L, Elsinga G, Italiaander R, Brouwer A. Presence of SARScoronavirus-2 RNA in sewage and correlation with reported COVID-19 prevalence in the early stage of the epidemic in the Netherlands. Environ Sci Technol Lett. Published online May 20, 2020. https://doi.org/10.1021/acs.estlett.0c00357

25. Nipissing First Nation. Community Notice: COVID-19 Detected in First Wastewater Sample. Nipissing First Nation; 2021. https://www.nfn.ca/wp-content/ uploads/2021/01/20210123-COVID-19-in-WW-Test.pdf

26. Bickford P. Signal of Covid-19 disappears from wastewater in Hay River. Published January 25, 2021. https://www.nnsl.com/nwtnewsnorth/signal-of-covid-19disappears-from-wastewater-in-hay-river/

27. Olesen SW, Imakaev M, Duvallet C. Applications of wastewater-based epidemiology as a leading indicator for COVID-19. Published online April 1, 2021. https://arxiv. org/abs/2104.00684

28. Health and Social Services. Five positive COVID-19 diagnoses identified in 
Yellowknife. https://www.hss.gov.nt.ca/en/newsroom/five-positive-covid-19diagnoses-identified-yellowknife

29. Naughton CC, Roman FA, Alvarado AGF, et al. Show us the data: global COVID-19 wastewater monitoring efforts, equity, and gaps. medRxiv. Published online March 17, 2021. https://doi.org/10.1101/2021.03.14.21253564

30. Wolfe MK, Archana A, Catoe D, et al. Scaling of SARS-CoV-2 RNA in settled solids from multiple wastewater treatment plants to compare incidence rates of laboratory-confirmed COVID-19 in their sewersheds. Environ Sci Technol Lett. 2021;8(5):398-404. https://doi.org/10.1021/acs.estlett.1c00184

31. The University of Arizona. WEST monitors wastewater to keep campus safe. Arizona University West Center. Published August 24, 2020. https://west.arizona. edu/news/2020/08/west-monitors-wastewater-keep-campus-safe

32. Peiser J. The University of Arizona says it caught a dorm's covid-19 outbreak before it started. Its secret weapon: Poop. The Washington Post. Published August 28, 2020. https://www.washingtonpost.com/nation/2020/08/28/arizonacoronavirus-wastewater-testing/

33. The University of Arizona. Wastewater testing at UArizona stops coronavirus spread; garners national attention. Arizona University West Center. Published August 31, 2020. https://west.arizona.edu/news/2020/08/wastewater-testinguarizona-stops-coronavirus-spread-garners-national-attention

34. Heaton KW, Radvan J, Cripps H, Mountford RA, Braddon FE, Hughes AO. Defecation frequency and timing, and stool form in the general population: a prospective study. Gut. 1992;33(6):818-824. https://doi.org/10.1136/gut.33.6.818

35. Walter S, Hallböök O, Gotthard R, Bergmark M, Sjödahl R. A Population-based Study on Bowel Habits in a Swedish Community: Prevalence of Faecal Incontinence and Constipation. Scand J Gastroenterol. 2002;37(8):911-916. https://doi. org/10.1080/003655202760230865

36. Walker AR, Walker BF. Bowel behaviour in young black and white children. Arch Dis Child. 1985;60(10):967-970. https://doi.org/10.1136/adc.60.10.967

37. Han MS, Choi EH, Chang SH, et al. Clinical Characteristics and Viral RNA Detection in Children With Coronavirus Disease 2019 in the Republic of Korea. JAMA Pediatr. Published online August 28, 2020. https://doi.org/10.1001/ jamapediatrics.2020.3988

38. Qiu H, Wu J, Hong L, Luo Y, Song Q, Chen D. Clinical and epidemiological features of 36 children with coronavirus disease 2019 (COVID-19) in Zhejiang, China: an observational cohort study. Lancet Infect Dis. 2020;20(6):689-696. https://doi. org/10.1016/S1473-3099(20)30198-5

39. Yonker LM, Neilan AM, Bartsch Y, et al. Pediatric SARS-CoV-2: Clinical Presentation, Infectivity, and Immune Responses. J Pediatr. 2020;0(0). https://doi.org/10.1016/j. jpeds.2020.08.037

40. Thompson JR, Nancharaiah YV, Gu X, et al. Making waves: wastewater surveillance of SARS-CoV-2 for population-based health management. Water Res. 2020;184:116181. https://doi.org/10.1016/j.watres.2020.116181

41. ILandgraff C, Wang LYR, Buchanan C, et al. Metagenomic sequencing of municipal wastewater provides a near-complete SARS-CoV-2 genome sequence identified as the B.1.1.7 variant of concern from a Canadian municipality concurrent with an outbreak. medRxiv. Published online March 17, 2021:2021.03.11.21253409. https://doi.org/10.1101/2021.03.11.21253409 
42. Matrajt G, Lillis L, Meschke JS. Review of methods suitable for environmental surveillance of Salmonella Typhi and Paratyphi. Clin Infect Dis. 2020;71(Supplement_2):S79-S83. https://doi.org/10.1093/cid/ciaa487

43. Hutinel M, Huijbers PMC, Fick J, Åhrén C, Larsson DGJ, Flach C-F. Population-level surveillance of antibiotic resistance in Escherichia coli through sewage analysis. Eurosurveillance. 2019;24(37):1800497. https://doi.org/10.2807/1560-7917. ES.2019.24.37.1800497

44. O'Keeffe J. Looking for COVID-19 clues in our sewers. Published January 15, 2021. https://ncceh.ca/content/blog/looking-covid-19-clues-our-sewers

45. Kantor RS, Nelson KL, Greenwald HD, Kennedy LC. Challenges in measuring the recovery of SARS-CoV-2 from wastewater. Environ Sci Technol. 2021;55(6):35143519. https://doi.org/10.1021/acs.est.0c08210

46. Tran HN, Le GT, Nguyen DT, et al. SARS-CoV-2 coronavirus in water and wastewater: A critical review about presence and concern. Environ Res. 2021;193:110265. https://doi.org/10.1016/j.envres.2020.110265

47. Canadian COVID-19 Wastewater Coalition. Phase 1 Inter-Laboratory Study: Comparison of Approaches to Quantify SARS-CoV-2 RNA in Wastewater. Canadian Water Network; 2020:25. https://cwn-rce.ca/wp-content/uploads/Covid-19-WWCoalition_Phase-1-Inter-Lab-Study-Outcomes_November-2020-1.pdf

48. Pecson BM, Darby E, Haas CN, et al. Reproducibility and sensitivity of 36 methods to quantify the SARS-CoV-2 genetic signal in raw wastewater: findings from an interlaboratory methods evaluation in the U.S. medRxiv. Published online November 4, 2020. https://doi.org/10.1101/2020.11.02.20221622

49. University of Windsor. Wastewater testing of campus residence leads to early disease detection. University of Windsor, Return to Campus. https:// www.uwindsor.ca/returntocampus/2021-03-29/wastewater-testing-campusresidence-leads-early-disease-detection

50. Wu F, Xiao A, Zhang J, et al. Wastewater Surveillance of SARS-CoV-2 across 40 U.S. states. medRxiv. Published online March 12, 2021:2021.03.10.21253235. https:// doi.org/10.1101/2021.03.10.21253235

51. Hart OE, Halden RU. Computational analysis of SARS-CoV-2/COVID-19 surveillance by wastewater-based epidemiology locally and globally: Feasibility, economy, opportunities and challenges. Sci Total Environ. 2020;730:138875. https://doi. org/10.1016/j.scitotenv.2020.138875

52. Kitajima M, Sassi HP, Torrey JR. Pepper mild mottle virus as a water quality indicator. Npj Clean Water. 2018;1(1):19. https://doi.org/10.1038/s41545-0180019-5

53. SARS-CoV-2 monitoring employing sewers: designing the EU sewer sentinel system for SARS-CoV-2 (EUS4). Published March 22, 2021. https://ec.europa.eu/ jrc/en/event/conference/eu4s-4th-town-hall-meeting

54. Research and innovation. EU to invest $€ 150$ million for research to counter coronavirus variants. European Commission - European Commission. Published February 17, 2021. https://ec.europa.eu/info/news/eu-invest-eu150-millionresearch-counter-coronavirus-variants-2021-feb-17_en

55. European Commission. Joint Research Centre. SARS-CoV-2 Surveillance Employing Sewage: Towards a Sentinel System. Publications Office; 2021. https:// op.europa.eu/en/publication-detail/-/publication/816cec6d-abbd-11eb-927e01aa75ed71a1/language-en 
56. Government Technology. HHS chooses biobot to test sewage for COVID-19 nationwide. GovTech. Published May 28, 2021. https://www.govtech.com/biz/ hhs-chooses-biobot-to-test-sewage-for-covid-19-nationwide

57. Water Research Australia. ColoSSoS - a world-first development and operation of sewage research for SARS-COV-2. WaterRA. Accessed August 17, 2020. https:// www.waterra.com.au/publications/latest-news/2020/colossos-a-a-world-firstdevelopment-and-operation-of-sewage-research-for-sars-cov-2/

58. Hrudey SE, Ashbolt NJ, Isaac-Renton JL, McKay RM. Wastewater-Based Epidemiology for SARS-CoV-2.; 2020:6. https://rsc-src.ca/sites/default/files/ Publication\%20\%2323\%20\%20-\%20EN\%20-\%20Epidimology_2.pdf

59. Raymond T. Premier's office calls Ottawa mayor "reckless and irresponsible" for opposing lockdown. CTV News. Published December 22, 2020. https://ottawa. ctvnews.ca/premier-s-office-calls-ottawa-mayor-reckless-and-irresponsible-foropposing-lockdown-1.5241594

60. Public Health Ontario. Focus on COVID-19 Wastewater Surveillance.; 2021. https://www.publichealthontario.ca/-/media/documents/ncov/phm/2021/04/ public-health-measures-wastewater-surveillance.pdf?la=en

61. Ontario Genomics. Sewage surveillance: detecting SARS-CoV-2 variants in wastewater across Ontario - Ontario Genomics. Published June 2, 2021. https:// www.ontariogenomics.ca/impact-stories/sewage-surveillance-detecting-sarscov-2-variants-in-wastewater-across-ontario/

62. Wang J, Yang W, Pan L, et al. Prevention and control of COVID-19 in nursing homes, orphanages, and prisons. Environ Pollut. 2020;266:115161. https://doi. org/10.1016/j.envpol.2020.115161

63. Territories G of N. Wastewater sampling to form COVID-19 "Early-Warning" system for Northwest Territories. https://www.gov.nt.ca/en/newsroom/wastewatersampling-form-covid-19-early-warning-system-northwest-territories

64. Daughton CG. Wastewater surveillance for population-wide Covid-19: The present and future. Sci Total Env. 2020;736:139631. https://doi.org/10.1016/j. scitotenv.2020.139631 